\title{
Endoscopic surgery for malignant sinonasal tumours: an eighteen year experience*
}

\section{Valerie J. Lund ${ }^{1}$ and William I. Wei²}

Royal National Throat Nose \& Ear Hospital, London, United Kingdom and University College London, London, United Kingdom

${ }^{2}$ Hong Kong Sanatorium and Hospital, Hong Kong
Rhinology 53: 204-211, 2015

DOl:10.4193/Rhino14.318

*Received for publication:

December 20, 2014

Accepted: February 11, 2015

\begin{abstract}
Introduction: Curative resection of malignant tumours of the skull base is increasingly undertaken endoscopically. Hitherto the diverse histology, rarity and long natural history have made it difficult to accrue statistically robust cohorts for comparison with conventional craniofacial resection. It is now possible to make such a comparison in a large personal cohort.
\end{abstract}

Method: Data on all cases of sinonasal malignancy undergoing endoscopic resection with curative intent over an eighteen year period were collected prospectively and analysed for survival and prognostic factors.

Results: There were 140 cases, 68 men and 72 women, aged 20-92 years (mean 63 yrs). Follow-up ranged from 6-184 months (mean 60 months). Eighteen different histopathologies were represented with olfactory neuroblastoma (36), malignant melanoma (33) and adenocarcinoma (19) being the commonest. Additional radiotherapy was given in 95 cases and chemotherapy in 49. Overall survival is $84 \%$ at 5 years and $69 \%$ at 10 years. Overall disease-free survival was $77 \%$ at 5 years and $56 \%$ at ten. Overall and disease-free survival at 5 (and 10) years is, respectively, $97 \%$ and $90 \%$ for olfactory neuroblastoma, $79 \%$ and $68 \%$ for adenocarcinoma and $56 \%$ and $39 \%$ for malignant melanoma.

Conclusion: These results show that endoscopic resection is an alternative to conventional craniofacial resection in selected cases.

Key words: endoscopic skull base surgery, anterior skull base, esthesioneuroblastoma

\section{Introduction}

An endoscopic approach has become the standard of care for the majority of conditions requiring surgery both within the nose and sinuses and at the interfaces with the orbit and skull base. Whilst the use of endoscopic techniques for benign neoplasia were well established, their application to malignant tumours remained controversial mainly due to concerns that the ability to undertake an adequate resection might be compromised. This combined with the rarity of these tumours, occurring in $<1 / 100,000$ of the population, their diverse histologies, variable natural histories and ability to recur many years later have made it difficult to accrue large series with sufficient follow-up to make statistically robust comparisons with 'gold standard' treatments such conventional craniofacial resection. However, it is clear from the literature ${ }^{(1,2)}$ that this situation is changing and there are a number of centres where experience with both endoscopic techniques and sinonasal malignancy have facilitated prospective collection of data on cohorts of reasonable size for such assessment. This paper documents one such cohort.

Diagnosis is often made late because these tumours are asymptomatic or produce non-specific symptoms in their early stages and although there are several staging systems, none are ideal or universally utilised. However, generally stage at presentation is highly predictive of survival ${ }^{(2,3)}$ and despite maximum treatment of the primary tumour, local, regional and distant metastases can occur and may do so after many years. 


\section{Materials and methods}

Data collection

This study considers all patients with $>6$ months follow-up undergoing endoscopic resection with curative intent for sinonasal malignancy in a single centre by the first author over an 18-year period, from 1996 to 2014, complete data on whom have been collected prospectively by the author. The diagnosis was confirmed by dedicated head and neck histopathologists, expert in sinonasal neoplasia. All patients underwent preoperative contrast-enhanced computerized tomography (CT) and magnetic resonance imaging (MRI) enhanced with gadolinium diethylenetriamine penta-acetic acid contrast of the nose, paranasal sinuses, entire intracranial compartment and neck together with imaging of the thorax and abdomen. Regular postoperative follow-up was undertaken using a rigorous protocol (1). Age, sex, stage, surgical management, pre- and post-operative treatment, recurrence and survival data were recorded.

\section{Treatment}

All patients deemed suitable after multi-disciplinary pre-operative assessment, underwent endoscopic surgery with curative intent, details of which have been previously published ${ }^{(4)}$ (Table 1) Wide-field mucosal clearance was performed according to the extent of disease, with resection of skull base whenever this was involved or adjacent to the tumour. If skull base was involved, adjacent dura was resected and in cases of olfactory neuroblastoma or when dura was affected, the olfactory bulbs and tracts were removed. Intracranial spread was divided into 3 groups: 1) tumour adjacent to or involving the bone of the skull base; 2 ) tumour adjacent to \pm microscopic involvement of the dura which was resected or 3) tumour extending through the full thickness of the dura into the anterior cranial fossa.

Orbital periosteum was resected endoscopically if adjacent to or involved by tumour. Orbital involvement was divided into 3 groups: 1) none where the lamina remained intact; 2) tumour adjacent to \pm microscopic involvement of orbital periosteum when periosteum was resected or 3) spread of tumour through the full thickness of the orbital periosteum confirmed on histological examination after resection of it and adjacent fat.

When deemed necessary, standard post-operative 3-field external beam radiotherapy to a dose of 55-65 Gy was given between 1996-2009. Since 2010, IMRT has been employed. The decision to give radiotherapy was based on previous published clinical experience regarding the histopathology together with adjuvant mainly cisplatin-based chemotherapy in selected cases. Specifically radiotherapy has been shown to be of significant benefit in olfactory neuroblastoma, squamous cell carcinoma, SNUC and rhabdomyosarcoma ${ }^{(2,3)}$ but of less proven value in adenocarcinoma, malignant melanoma and chondrosarcoma
Table 1. Potential limitations for an entirely endoscopic approach where a combined conventional and endoscopic CFR may be considered.

Tumour through dura infiltrating falx/sagittal sinus and/or frontal lobe

Tumour extension lateral to mid-point of orbit

Wide dural involvement, especially in proximity to optic chiasm

And/or orbital clearance

Tumour through orbital periosteum to involve orbital contents eg fat, muscle, neurovasculature

Type of malignancy eg type of tumour which can embolise within orbit such as squamous cell carcinoma

$(2,5,6)$.

\section{Statistical analysis}

Data was analysed using SPSS version 14.0 (IBM, Chicago, IL, USA) by the second author. Outcome measures were overall survival (OS) and disease free survival (DFS), also stratified by orbital and intracranial involvement. They were evaluated by KaplanMeier curves and statistical significance was calculated by the Mantel-Cox test. Cox regression analysis was used to identify any independent factors affecting overall survival. Univariate analysis included sex, previous radiotherapy, chemotherapy and/or surgery, subsequent radiotherapy, chemotherapy and/ or surgery, T staging, orbital and intracranial involvement and histological type of tumour.

\section{Results}

One hundred and forty patients were treated surgically with curative intent from 1996 - 2014, 68 male (49\%) and 72 female (51\%) aged 20-92 years (mean $63 \mathrm{yrs).}$

Eighteen different histopathologies were represented with olfactory neuroblastoma (ON) (36 cases), mucosal malignant melanoma (MMM) (33 cases) and adenocarcinoma (AC) (19 cases) being the commonest (Table 2). The site of origin was predominantly nasal cavity ( 89 cases: $64 \%$ ) or ethmoids ( 43 cases: $31 \%$ ) with 2 cases each arising in and confined to the frontal or sphenoid and 4 in the maxillary sinus, all regarded as amenable to endoscopic excision.

Table 3 shows T staging when applied to all tumours, the majority being $\mathrm{T} 1 / \mathrm{T} 2$. Only one patient had neck involvement at presentation. None presented with distant metastases. In keeping with the most recent AJCC staging ${ }^{(7)}$, all melanomas have been staged as T3 or greater. However, it should be noted that the AJCC staging is not designed for application to nonepithelial tumours or those of lymphoid tissue, soft tissue, bone 
Table 2. ESS for Malignant Sinonasal Tumours Personal series 1996-2014.

\begin{tabular}{|l|ccccc|}
\hline Histology & N & $\begin{array}{c}\text { Range of FU } \\
\text { (months) }\end{array}$ & Mean & SE \\
\hline Olfactory neuroblastoma & 36 & $6-162$ & 77.6 & 10.7 \\
\hline Malignant melanoma & 33 & $6-167$ & 68.6 & 7.6 \\
\hline Adenocarcinoma & 19 & $2-99$ & 33.1 & 5.1 \\
\hline Other histologies & 52 & $3-184$ & 59.7 & 6.1 \\
\hline Squamous cell carcinoma & 9 & & & \\
\hline Haemangiopericytoma & 8 & & & \\
\hline Chondrosarcoma & 8 & & & \\
\hline Malignant schwannona & 5 & & & \\
\hline Sinonasal undifferentiated & 5 & & & \\
\hline carcinoma & 19 & & & \\
\hline Lymphoma & 1 & & & \\
\hline Rhabdomyosarcoma & 2 & & \\
\hline Leiomyosarcoma & 2 & & \\
\hline Transitional cell carcinoma & 2 & & \\
\hline Cylindric cell carcinoma & 2 & & \\
\hline Ewing's sarcoma & 1 & & \\
\hline Plasmacytoma & 1 & & \\
\hline Malignant fibrous & & & & \\
\hline histiocytoma & & & & \\
\hline Neuroendocrine carcinoma & 1 & & & \\
\hline Adlow up & & & & \\
\hline
\end{tabular}

or cartilage. This would include 38 patients in this cohort who are considered separately in terms of survival.

Orbital periosteum was resected and repaired in 12 (9\%). In this series no involvement of muscle was encountered on frozen section and thus no formal orbital clearance was undertaken. Dura was resected and a skull base repair undertaken in 35 (25\%) cases which included 20 olfactory neuroblastomas for purposes of staging.

Prior to referral to our unit, 115 patients were untreated and 25 patients had received and failed previous treatment. Eight patients had been treated with radiotherapy and 9 had received both chemotherapy and radiotherapy. Eleven patients had un-
Table 3. T staging of entire cohort ${ }^{(7)}(n=140)$.

\begin{tabular}{|c|c|c|}
\hline & $\mathbf{N}$ & $\%$ \\
\hline $\mathrm{T} 1$ & 57 & 41 \\
\hline $\mathrm{T} 2$ & 27 & 19 \\
\hline T3 & 41 & 29 \\
\hline $\mathrm{T} 4$ & 17 & 11 \\
\hline
\end{tabular}

All $\mathrm{N}$ are $\mathrm{MO}$ except one T3.

dergone previous surgery, either via lateral rhinotomy (2 cases) midfacial degloving ( 2 patients), endoscopically (5 patients) or a craniotomy (2 patients). Of these 11, 3 had also received radiotherapy. Previous treatment did not adversely affect subsequent outcome ( $p>0.1$ for all treatments) though as the numbers are small, this may be a type II error.

Hospital stay ranged from 3-8 days, mean 4.5 days and most patients were readmitted as a daycase for removal of packing at 10-12 days. Follow-up ranged from $6-210$ months (mean 60 months). The first type of tumour treated endoscopically was malignant melanoma from 1996 onwards but from 1998 all histologies were considered. This is reflected in the numbers performed each year with 8 cases being undertaken between 1996-2000, 41 between 2001-2005, 54 between 2006-2010 and 37 between 2011 and mid-2014 when this analysis was undertake equating to 10 cases each year during the last decade.

There were few peri- or post-operative complications and all were resolved without significant morbidity. These comprised three cases (2\%) who required further packing for bleeding in the immediate post-operative period and cerebrospinal fluid leakage ( 3 cases $=2 \%$ ), which unusually occurred 9 months, 1 and 4 years after initial treatment. These were successfully treated endoscopically. Epiphora occurred in 8 cases (6\%), all of whom had received combined surgery and radiotherapy, and six of whom underwent successful endoscopic dacrocystorhinostomies. One patient developed pituitary insufficiency 5.5 years after combined surgery and radiotherapy.

Ninety-five (68\%) patients received post-operative radiotherapy and 49 (34\%) had additional chemotherapy. The radiotherapy was given to 33 patients with ON, 20 patients with MMM, 14 adenocarcinomas and 27 of the 52 other individuals. This was combined with chemotherapy in $25 \mathrm{ONs}, 4$ cases each of squamous cell carcinomas, MMM and sinonasal undifferentiated carcinoma (SNUC), lymphoma, 2 cases each of poorly differentiated adenocarcinoma, rhabdomyosarcoma and leiomyosarcoma, one 
Table 4. Five and 10 year \% survival for whole group and individual histologies.

\begin{tabular}{|lcccccc|}
\hline & N & \multicolumn{2}{c}{ Overall survival } & \multicolumn{2}{c|}{$\begin{array}{c}\text { Disease-free } \\
\text { survival }\end{array}$} \\
& & $\mathbf{5 y r}$ & $\mathbf{1 0 y r}$ & $\mathbf{5 y r}$ & $\mathbf{1 0 y r}$ \\
\hline Whole group & 140 & $84 \%$ & $69 \%$ & $77 \%$ & $56 \%$ \\
\hline Olfactory neuroblastoma & 36 & $97 \%$ & $97 \%$ & $90 \%$ & $90 \%$ \\
\hline Adenocarcinoma & 19 & $79 \%$ & $72 \%$ & $68 \%$ & $61 \%$ \\
\hline Malignant melanoma & 33 & $56 \%$ & $0 \% *$ & $39 \%$ & $0 \% *$ \\
\hline
\end{tabular}

*Survival for MM at 91 months 24\% (overall) and 17\% (DFS)

case each of plasmacytoma and Ewing's sarcoma.

Fifteen cases (11\%) have undergone additional endoscopic surgery, in 6 cases on multiple occasions, for local recurrence during the course of follow-up (MMM (11 cases), ON ( 2 cases), AC ( 2 cases) and chondrosarcoma ( 1 case)). These occurred within the surgical cavity at a wide range of locations of which 2 involved the skull base (ON [1], MM [1]) and one the orbit (CS). Local recurrence occurred within the first 12 months in most cases but one ON recurred at 39 months and one AC at 8 years. Four cases were converted to formal craniofacial resection at $6,10,12$ and 13 months for ON ( 2 cases), AC and transitional cell carcinoma due to dural/intracranial recurrence deemed to be beyond what could be safely encompassed by an entirely endoscopic approach.

Routine initial staging and regular follow-up MRI included the neck, together with ultrasound and fine needle aspiration for any cervical lymphadenopathy. As a consequence, nine patients have had unilateral ipsilateral neck dissections performed ( $1 \mathrm{ON}$ prior to ESS and 4 other ON cases at 10, 14, 18 and 26 months and 4 cases of MMM between 5 and 50 months) and one patient with ON had bilateral neck dissections (ipsilateral at 2 and contralateral at 4 years) after the initial ESS. Of these patients, two thirds had no recurrent disease at the primary site. Additional radiotherapy to the neck was given to 4 patients. Distant metastases occurred in 13 patients, 7 MMMs, 2 poorly differentiated $\mathrm{ACs}$ and one case each of transitional cell carcinoma, adenoid cystic, ON and leiomyosarcoma; 11 have succumbed and 2 are alive but expected to die of their disease, both MMMs.

Ninety six patients are alive and well, 16 alive with recurrence, 20 dead of disease and 5 have died of intercurrent disease. Three have been lost to follow-up. Those alive with local recurrence comprise MMM (9), AC (2), ON (2), chondrosarcoma (2) and squamous cell carcinoma (1). Thirteen patients with MMM, 2 ACs and 1 each of transitional cell carcinoma, ON, adenoid cystic carcinoma, SNUC and leiomyosarcoma have died of disease. Of those 15 undergoing further endoscopic surgery for local recurrence, control thus far has been achieved in 10 (but not in 5 of the melanomas). Of those undergoing formal craniofacial resection, 3 have survived but the patient with transitional carcinoma succumbed 11 months later. Of those undergoing neck dissection, all ON patients are alive but all MMM patients have died.

Overall crude survival is $84 \%$ at 5 years and $69 \%$ at 10 years. Overall disease-free survival was $77 \%$ and $56 \%$ at 5 and 10 years. Overall and disease-free survival at 5 years is, respectively, $97 \%$ and $90 \%$ for ON, $79 \%$ and $68 \%$ for AC and $56 \%$ and $39 \%$ for MMM (Table 4). Mean survival times are shown in Table 5.

When overall survival and disease-free survival for $\mathrm{ON}, \mathrm{AC}$, $M M M$ and the rest of the group are compared, ON has the best outcome, followed by AC, the miscellaneous group of mainly non-epithelial histologies whilst MMM, as anticipated comes last (Figures 1-4).

Table 5. Mean overall and disease-free survival for whole group and individual histologies.

\begin{tabular}{|c|c|c|c|c|c|c|c|}
\hline & $\mathbf{N}$ & $\begin{array}{c}\text { Mean overall } \\
\text { survival (months) }\end{array}$ & SE & $\mathbf{C l}$ & $\begin{array}{l}\text { Mean disease-free } \\
\text { survival (months) }\end{array}$ & SE & $\mathbf{C l}$ \\
\hline Whole group & 140 & 141 & 7.21 & 26.7-1551 & 120 & 7.5 & 105.1-134.6 \\
\hline Olfactory neuroblastoma & 36 & 162 & 4.5 & $153.4-171.2$ & 150 & 7.9 & $134.2-165.2$ \\
\hline Adenocarcinoma & 19 & 130 & 13.5 & 103.5-156.7 & 118 & 14.1 & $90.5-145.8$ \\
\hline Malignant melanoma & 33 & 58 & 8.0 & 42.4-73.9 & 47 & 7.3 & $32.7-61.4$ \\
\hline
\end{tabular}


Survival Function

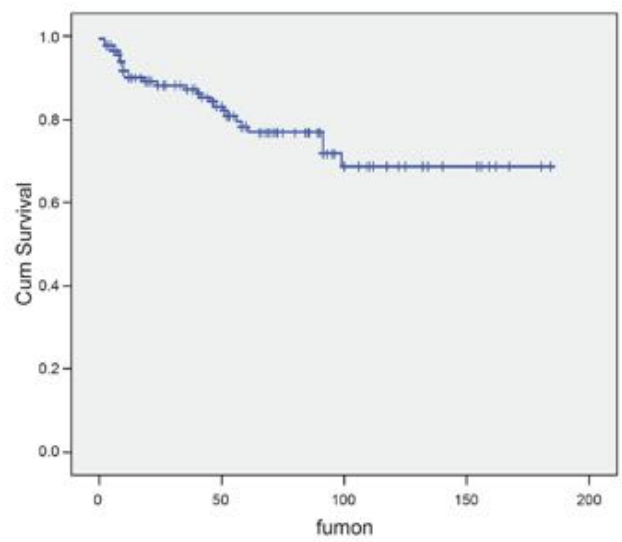

Figure 1. Kaplan-Meier graph of overall survival of 140 cases of sinonasal malignancy.

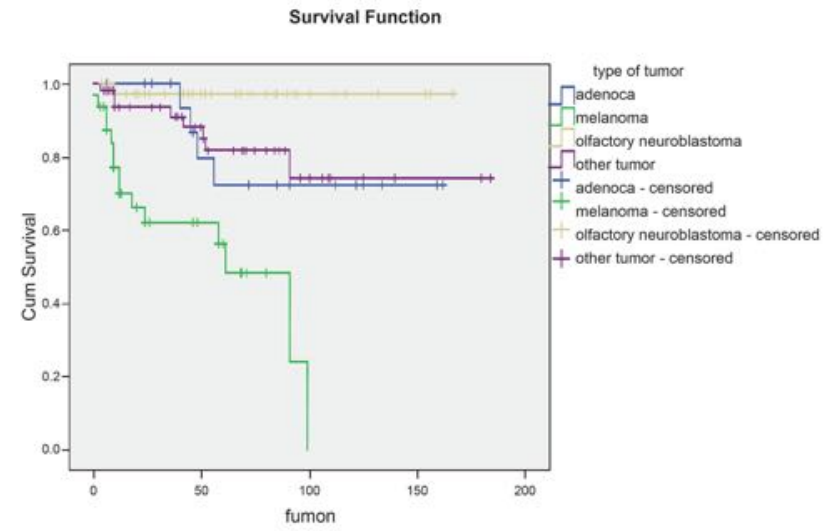

Figure 3. Kaplan-Meier graph of overall survival comparing adenocarcinoma, malignant melanoma, olfactory neuroblastoma and the remaining other tumour histologies.

A Cox regression analysis on the entire cohort shows T stage to be the most significant prognostic factor affecting overall survival followed by previous surgery and orbital involvement (Mantel Cox $p=0.002$, respectively). In addition to T stage, for $\mathrm{AC}$ and $\mathrm{ON}$ both extent of intracranial and orbital involvement are significant but for MMM none of these factors are significant.

\section{Discussion}

Since endoscopic techniques have been applied with curative intent, there have been many publications though the vast majority have been characterised by small numbers, mixed histologies and short follow-up. In 2010, the European Position Paper on the Endoscopic Management of Tumours of the Nose, Paranasal Sinuses and Skull Base ${ }^{(1)}$ summarised the literature to date and called for collaboration in the prospective collection of data on these rare cases. Although this has met with some success,

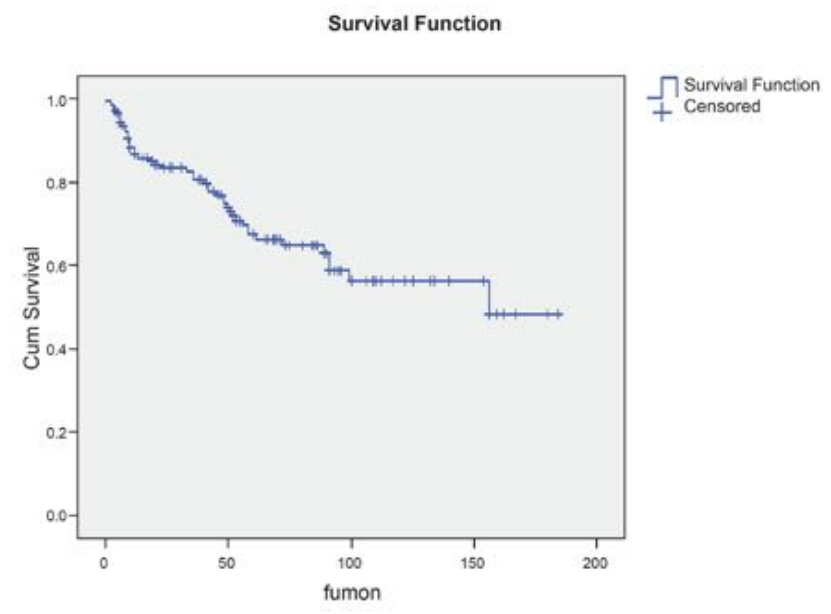

Figure 2. Kaplan-Meier graph of disease-free survival of 140 cases of sinonasal malignancy.

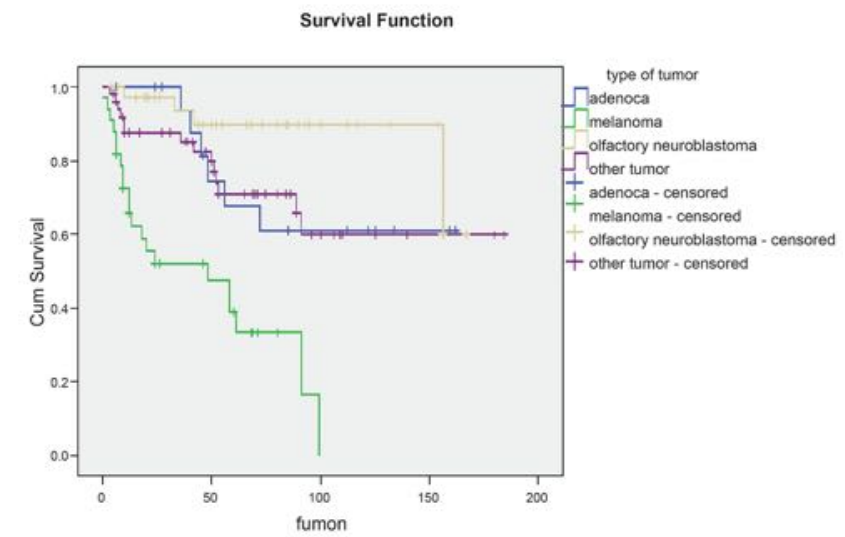

Figure 4. Kaplan-Meier graph of disease-free survival comparing adenocarcinoma, malignant melanoma, olfactory neuroblastoma and the remaining other tumour histologies.

there are still relatively few publications of large cohorts with long-term follow-up. This paper builds on previous publications from a single centre, which have also allowed comparison with alternative open approaches such as craniofacial resection (CFR) and lateral rhinotomy. In keeping with these and the experience of other units ${ }^{(4,8-13)}$, one may conclude that endoscopic approaches may be undertaken in cases of appropriate histology and extent with the expectation of equivalent results and in many cases, reduced morbidity and hospital stay.

This cohort, in common with others, comprises a wide range of histologies but is sufficiently large to include larger groups of individual tumour types such as ON, AC and MMM, which may be analysed separately and demonstrate that ON has the most favourable outcome and MMM the worst. In a recent study in which ON treated with endoscopic surgery was compared to 
CFR, there was significantly improved OS in the endoscopic group with 5 -year survival of $100 \%$ compared to $76.5 \%$ in the CFR group directly attributable to the less advanced disease being treated endoscopically, as might be expected ${ }^{(3)}$. When tumours of similar stage were compared, survival was equivalent. A meta-analysis of 361 cases of ON reported a significantly higher survival rate for endoscopic versus open surgery, related to tumour extent ${ }^{(14)}$. Similarly in a series of 35 patients, Song et al reported significantly higher 5-year disease free survival in those who underwent endoscopic resection when compared to open surgery ${ }^{(15)}$.

The same situation has been demonstrated for $A C$ and MMM in literature reviews and a number of recent endoscopic series ${ }^{(5,8,16-}$ 21). However, in the case of sinonasal MMM, endoscopic resection may even confer survival benefit irrespective of tumour extent (6). Since 1996, endoscopic resection had been employed irrespective of tumour extent yet when this was compared to open approach resection, there was a significantly improved overall survival in the endoscopic patients (Mantel-Cox $p=0.013$ ). They had a longer median overall survival (59 month compared to 18 months for open approaches), longer local control (50 months $v$ 18 months and longer median disease free survival (36 months $v$ months versus 16 months). The precise explanation for this difference is unclear but may relate to the reduced morbidity of the endoscopic surgery compared to open procedures in elderly patients with an immunologically capricious disease and has been observed by others ${ }^{(22)}$.

Generally the complication rate and overall morbidity of endoscopic procedures compares favorably with the open procedures such as CFR ${ }^{(3,11,23,24)}$ and those that do occur, such as CSF leaks or epiphora are readily amenable to endoscopic repair. The late development of a CSF leak in 3 of our cases is unusual as only one occurred in association with a recurrence. However, late failure of endoscopic skull base repair has been reported, albeit rarely ${ }^{(25)}$. Advances in endoscopic techniques allow repair of large anterior skull base defects and are associated with low complication rates even though the extent of surgery is comparable to that of open procedures ${ }^{(1,4,26-28)}$. It is also possible to commence additional radiotherapy and/or chemotherapy within a few weeks of surgery which may be considered advantageous ${ }^{(29,30)}$. However, the use of radiotherapy may lead to a loss of smell which otherwise would be less affected than with conventional CFR ${ }^{(31)}$.

In ON, radiotherapy has been shown to reduce local recurrence and improve prognosis so is recommended in all cases, irrespective of extent ${ }^{(32-35)}$. It is also well-established in squamous cell carcinoma, SNUC and rhabdomyosarcoma but evidence for its role in other tumours such as AC and MMM ${ }^{(5,6,22,36,37)}$ is less robust. Notwithstanding this, post-operative radiotherapy is often given and there is increasing use of intensity-modulated radiotherapy (IMRT) in the expectation of reducing damage to adjacent structures ${ }^{(38,39)}$.

The TNM staging system may be applied to sinonasal tumours but it was largely designed for squamous cell carcinoma and modifications of this and other systems have been proposed for individual tumors such as $\mathrm{ON}$ and $\mathrm{MMM}^{(2,7,40,41)}$. The 7 th edition of the AJCC Cancer staging manual recommends all mucosal malignant melanomas are staged $\mathrm{T} 3$ or greater in recognition of their aggressive behaviour ${ }^{(7)}$. Thus nearly two thirds of our patients are smaller tumours i.e. T1 and T2, and the majority of the T3 tumours are MMMs in contrast to some other series of more extensive lesions.

Stage at presentation has been shown to be highly predictive of survival and the outcomes from our series support this with the exception of malignant melanoma $(3,5,6,11,21,24,42,43)$. In ON a meta-analysis of 26 studies (390 patients) showed significantly better 5-year survival for Kadish stage A tumours (72\%) compared to stage B (59\%) or stage C $(47 \%)^{(32)}$. Lund et al. reported a $61 \%$ 5-year and 42\% 10-year overall survival in 42 patients who underwent CFR for ON ${ }^{(33)}$. The vast majority of patients in this series are T1-3 and would therefore be anticipated to do as well as those undergoing CFR, as has been demonstrated in this analysis.

All patients with the exception of one $\mathrm{ON}$ in this series were NO at presentation, though 9 developed cervical lymphadenopathy during follow-up. Cervical metastases are reported in approximately $10 \%$ of cases of sinonasal malignancy and confer a significantly worse prognosis. This was most common in ON and $M M M$ and for this reason, imaging of the neck is undertaken pre- and post-operatively as part of routine follow-up ${ }^{(44)}$. Distant metastases to brain/dura, lung, liver, bone, spinal cord, liver and skin are rare at presentation but can occur, especially in the terminal stages of disease ${ }^{(2)}$.

From a surgical perspective, the degree of intracranial extension and orbital involvement have been shown to be independent prognostic factors ${ }^{(24)}$ and are also the determinants of whether an entirely endoscopic approach is possible. However, it should be noted that a combination of endoscopic and open techniques, is an alternative in tumours with moderate intracranial involvement ${ }^{(45,46)}$.

Clearly it is still necessary to retain the open approaches in one's armamentarium for those tumours which present with extensive involvement of the orbit and intradural compartment or for those, as in 4 cases in this series, who develop further disease not amenable to a further endoscopic removal and who subse- 
quently underwent conventional CFR.

Sinonasal malignancy may recur many years after initial treatment. The longest time to local recurrence in this cohort was 8 years (a case of AC) and in our entire series of ON 233 months (2). This was not apparent during the early reported series of endoscopic patients but now that cohorts with follow-up of substantial duration are available, up to 16 years in this series, this problem can be shown to occur, confirming that life-long follow-up is required irrespective of the treatment.

This series supports the contention that endoscopic resection offers equivalent results to conventional external procedures. The lower complication rate and peri-operative morbidity together with shorter hospital stay make an endoscopic ap- proach which adheres to oncologic principles a more than acceptable alternative in appropriate cases. However, all other aspects of pre- and post-operative care remain unchanged and life-long follow-up is mandatory with clinical examination and MRI. Prospective collection of data is also fundamental to our understanding of the natural history and management of these rare diseases.

\section{Author contributions}

VJL undertook the surgery and WIW undertook the statistical analysis of data. Both wrote the paper.

\section{Conflict of interest}

There are no conflicts of interest to declare, financial or otherwise. No funding was received.

\section{References}

1. Lund VJ, Stammberger H, Nicolai $P$, Castelnuovo P et al. European Position Paper on Endoscopic Management of the Nose, Paranasal Sinuses and Skull Base. Rhinology. 2010; Supplement 22: 1-144.

2. Lund VJ, Howard D, Wei W. Tumours of the nose, sinuses and nasopharynx. Thieme 2014. 1-595.

3. Rimmer J, Lund V, Beale T, Howard D, Wei W. Olfactory neuroblastoma - a 35 year experience and suggested follow-up protocol Laryngoscope. 2014; 124: 1542-1549.

4. Lund VJ. Endoscopic Resection of malig nant tumors of the nose and sinuses. Am J Rhinol. 2007: 21: 89-94.

5. Lund VJ, Chisholm E, Takes R, et al. Evidence for treatment strategies in sinonasal adenocarcinoma. Head Neck. 2012; 34: 1168-1178.

6. Lund VJ, Chisholm E, Howard D, Wei W Sinonasal melanoma: a review of 115 cases assessing outcomes of surgery, postoperative radiotherapy and endoscopic resection. Rhinology. 2012; 50: 203-210.

7. Edge SB, Compton CC, Fritz AG, et al. AJCC cancer staging manual (7th ed). New York: Springer, 2011.

8. Nicolai P, Castelnuovo P, Lombardi D, et al Role of endoscopic surgery in the management of selected malignant epithelial neoplasms of the naso-ethmoidal complex. Head Neck 2007; 29: 1075-1082.

9. Snyderman C, Carrau R, Kassam A, et al Endoscopic skull base surgery: Principles of endonasal oncological surgery. Journal of Surgical Oncology 2008;97:658-664

10. Eloy J, Vivero R, Hoang K, et al. Comparison of transnasal endoscopic and open craniofacial resection for malignant tumors of the anterior skull base. Laryngoscope. 2009; 119: 834-840.

11. Folbe A, Herzallah I, Duvvuri U, et al. Endoscopic endonasal resectio of esthesioneuroblastoma: A multicenter study. Am J Rhinol Allergy. 2009; 23: 91-94.

12. Jardeleza C, Seiberling K, Floreani S,
Wormald PJ. Surgical outcomes of endoscopic management of adenocarcinoma of the sinonasal cavity. Rhinology. 2009; 47: 354-361.

13. Ensenat J, de Notaris M, Sanchez M, et al. Endoscopic endonasal surgery for skull base tumours: technique and preliminary results in a consecutive case series report. Rhinology. 2013; 51: 37-46.

14. Devaiah AK, Andreoli MT. Treatment of esthesioneuroblastoma: a 16-year metaanalysis of 361 patients. Laryngoscope. 2009; 119: 1412-1416.

15. Song CM, Won T-B, Lee CH, Kim D-Y, Rhee C-S. Treatment modalities and outcomes of olfactory neuroblastoma. Laryngoscope. 2012; 122: 2389-2395.

16. Goffart $Y$, Jorissen M, Daele J, et al. Minimally invasive endoscopic management of malignant sinonasal tumours. Acta Otorhinolaryngol Belg. 2000; 54: 221-232.

17. Hanna E, DeMonte F, Ibrahim S, et al. Endoscopic resection of sinonasal cancers with and without craniotomy: oncologic results. Arch Otolaryngol Head Neck Surg 2009; 135: 1219-1224.

18. Moreno MA, Hanna EY. Management of mucosal melanomas of the head and neck: did we make any progress? Curr Opin Otolaryngol Head Neck Surgery 2010; 18: 101-106.

19. Roth TN, Gengler C, Huber GF, Holzmann D. Outcome of sinonasal melanoma: Clinical experience and review of the literature. Head Neck. 2010; 32: 1385-1392.

20. Van Gerven L, Jorissen M, Nuyts S, et al. Long-term follow-up of 44 patients with adenocarcinoma of the nasal cavity and sinuses primarily treated with endoscopic resection followed by radiotherapy. Head Neck. 2010; 32: 1-8.

21. Antognoni P, Turri-Zanoni M, Gottardo S, et al. Endoscopic resection followed by adjuvant radiotehrapy for sinonasal intestinaltype adenocarcinoma: retrospective analysis of 30 consecutive patients. Head Neck epub 2014 DOI 10.1002/hed.23660

22. Moreno MA, Roberts DB, Kupferman MEet al. Mucosal melanoma of the nose and paranasal sinuses, a contemporary experience from the M. D. Anderson Cancer Center. Cancer; 2010; 116: 2215-2223.

23. Ganly I, Patel SG, Singh B, et al. Craniofacial resection for malignant paranasal sinus tumors: Report of an International Collaborative Study. Head Neck. 2005; 27: 575-584.

24. Howard DJ, Lund VJ, Wei WI. Craniofacial resection for sinonasal neoplasia - a twenty-five year experience. Head Neck. 2006; 28: 867-873.

25. Zuckerman J, Stankiewicz J, Chow J. Longterm outcomes of endoscopic repair of cerebrospinal fluid leaks and meningoencephalocoles. Am J Rhinol. 2005; 19: 582-587.

26. Walch C, Stammberger H, Anderhuber W, Unger F, Köle W, Feichtinger K. The minimally invasive approach to olfactory neuroblastoma: combined endoscopic and stereotactic treatment. Laryngoscope. 2000; 110: 635-640.

27. Castelnuovo P, Bignami M, Delù $G$, Battaglia P, Bignardi M, Dallan I. Endonasal endoscopic resection and radiotherapy in olfactory neuroblastoma: our experience. Head Neck 2007; 29: 845-850.

28. Nicolai P, Battaglia P, Bignami $M$, et al. Endoscopic surgery for malignant tumors of the sinonasal tract and adjacent skull base: a 10-year experience. Am J Rhinol. 2008; 22: 308-316.

29. Polin RS, Sheehan JP, Chenelle AG, et al. The role of preoperative adjuvant treatment in the management of esthesioneuroblastoma: the University of Virgina experience. Neurosurgery 1998; 42:1029-1037.

30. Nikapota AD, Sevitt T, Lund VJ, et al. Outcomes of radical conformal radiotherapy and concomitant cisplatin chemotherapy for olfactory neuroblastoma - review of a single centre experience. J Clin Oncol 2006; 24: 5555 . 
31. Bramerson A, Nyman J, Nordin S, Bende M Olfactory loss after head and neck cancer radiation therapy. Rhinology. 2013; 51: 206209.

32. Dulguerov P, Allal AS, Calcaterra TC. Esthesioneuroblastoma: a meta-analysis and review. Lancet. 2001; 2: 683-690.

33. Lund VJ, Howard D, Wei W, Spittle M. Olfactory neuroblastoma: past, present, and future? Laryngoscope. 2003: 113; 502-507.

34. Unger F, Haselsberger K, Walch C, Stammberger $H$, Papaefthymiou G. Combined endoscopic surgery and radiosurgery as treatment modality for olfactory neuroblastoma. Acta Neurochirurgica. 2005; 147: 595-602

35. Castelnuovo P, Bignami M, Delu G, et al. Endonasal endoscopic resection and radiotherapy in olfactory neuroblastoma. Head Neck. 2007; 29: 845-850.

36. Owens JM, Roberts DB, Myers JN. The role of postoperative adjuvant radiation therapy in the treatment of mucosal melanomas of the head and neck region. Arch Otolaryngol Head Neck Surg. 2003; 129: 864-868.

37. Clifton $N$, Harrison L, Bradley P, Jones $N$ Malignant melanoma of nasal cavity and paranasal sinuses: report of 24 patients and literature review. J Laryngol Otol. 2011; 125:
479-485.

38. Daly ME, Chen AM, Bucci MK, et al. Intensity-modulated radiation therapy for malignancies of the nasal cavity and paranasal sinuses. Int J Radiation Oncol Biol Physics. 2007; 67: 151-157.

39. Krengli M, Jereczek-Fossa BA, Kaanders JH, Masini L, Beldi D, Orecchia R. What is the role of radiotherapy in the treatment of mucosal melanoma of the head and neck? Crit Rev Oncol Hematol. 2008; 65: 121-128.

40. Kadish S, Goodman M, Wang CC. Olfactory neuroblastoma. A clinical analysis of 17 cases. Cancer. 1976; 37: 1571-1576.

41. Dulguerov $P$, Calcaterra $T$. Esthesioneuroblastoma: the UCLA experience 1970-1990. Laryngoscope. 1992; 102 843-849.

42. Choussy O, Ferron C, Vedrine PO, et al Adenocarcinoma of Ethmoid: a GETTEC retrospective multicenter study of 418 cases. Laryngoscope. 2008; 118: 437-443.

43. Jangard M, Hansson J, Ragnarsson-Olding B. Primary sinonasal malignant melanoma: a nationwide study of the Swedish population, 1960-2000. Rhinology. 2013; 51: 22-30.

44. Rinaldo A, Ferlito A, Shah AR, et al. Esthesioneuroblastoma and cervical lymph node metastases: clinical and therapeutic implications. Acta Otolaryngol. 2002; 122 215-221.

45. Thaler E, Kotapka M, Lanz D, Kennedy W. Endoscopically assisted anterior cranial skull base resection of sinonasal tumors. Am Rhinol Allergy. 1999; 13: 303-310.

46. Po-Wing Yuen A, Wah Fan Y, Fai Fung C, Ngai Hung K. Endoscopic-assisted cranionasal resection of olfactory neuroblastoma. Head Neck. 2005; 27: 488-493.

Valerie J. Lund

Royal National Throat, Nose \& Ear

Hospital

330 Gray's Inn Road

London WC1X 8DA

United Kingdom

Tel: +44-203-456 5197

Fax: +44- 207-8339480

E-mail:v.lund@ucl.ac.uk 